\title{
A WAY TO PREDICT PHYSICAL AND MECHANICAL PROPERTIES NANOFIBERS STRUCTURES THROUGH IMAGE ANALYSIS METHODS
}

\author{
${ }^{1}$ Lucie SVOBODOVÁ, ${ }^{2}$ Karel HAVLÍČEK, ${ }^{1}$ Totka BAKALOVA \\ ${ }^{1}$ Department of Material Science, Faculty of Mechanical Engineering, Technical University of Liberec, \\ Liberec, Czech Republic, EU, lucie.svobodova@tul.cz, totka.bakalova@tul.cz \\ 2Institute for Nanomaterials, Advanced Technology and Innovation, Technical University of Liberec, \\ Liberec, Czech Republic, EU, karel.havlicek@tul.cz
}

https://doi.org/10.37904/nanocon.2019.8553

\begin{abstract}
Nanofiber's morphology features (diameter, pore size, roughness) together with mechanical properties and biocompatibility are crucial for the evaluation of nanofibrous web's structures properties. Surface's morphology strongly affects among all the physical, mechanical, and biological properties of the web's structures. A detailed characterization of scanning electron microscopy images is decisive and widely used. Image analysis include most often the determination of fiber amount and their diameters on the monitored area; occasionally also the porosity and pore size determination; and very rarely the other parameters like linearity/curvature rating or orientation of fibers etc. Characterization by confocal microscopy is not yet commonly applied. However, the detailed observation of the surface and its evaluation bring many advantages, especially surface roughness determination but also the surface kurtosis and surface skewness etc. This work briefly summarizes the evaluation methods applicable for morphological characterization of nanofibers from SEM and confocal microscopy; and bringing closer insight for possible prediction of physical, mechanical, and biological properties of the nanofibers.
\end{abstract}

Keywords: Nanofiber characterization, image analysis, scanning electron microscopy, confocal microscopy

\section{INTRODUCTION}

The electrospinning process outcomes are a nonwoven web of differently oriented fibers with diameters in the nanometer scale. These nanofibers (NFs) have high specific surface area, large number of intra fibrous pores, mechanical properties superior to thicker fibers, so it is an excellent material for many applica-tions. The properties of NFs are mostly characterized through chemical composition, hydrophobicity, bioco-mpatibility, thermal and mechanical properties. However, the morphology (i.e. fiber orientation, diameter, pore size, roughness etc.) also affect the physical and mechanical properties of the webs [1]. Distribution of fiber morphology properties fluctuates on the methods and materials used. [2] A precise measurement of NF morphology based on SEM images is important. The articles describe structural parameters of NFs only briefly and only as a part of service methods. Only a few reports are focused on the theoretical appro-ach to calculate for example fiber diameter, orientation, length, pore size. [1,3-5] Morphological parameters show critical effect on the physical properties of NFs. [6] Internal architecture of NFs materials is an impor-tant attribute which predisposes them as supporting materials e.g. carriers for cell cultivation [7,8] (the scaffold requirements include biocompatibility, controlled porosity and permeability and suitable mechanical properties comparable to natural tissue) [4]; rough and smooth surfaces will encourage different types of cell adhesion [9]; preparation of the filter with well-defined pore dimensions improves filtering ability $[9,10]$. The aim of our investigation is to use image analysis methods for electrospun NF properties measurement from SEM images. The result is a more detailed examination of the NF webs, providing additional information that allows predicting the properties or behavior of materials and can improve the quality of final performance. 


\section{MATERIAL AND METHODS}

\subsection{Samples processing}

The polyvinylbutyral (PVB), Mowitals B $60 \mathrm{H}$, was obtained from Kuraray America, Inc. with the average molecular weight of $60000 \mathrm{amu}$. A $10 \mathrm{wt} \%$ spinning solution was prepared by dissolving the PVB polymer in ethanol, ratio 9:1 (90 g Et-OH : $10 \mathrm{~g} \mathrm{PVB}$ ). From the different concentrations of the solutions of polyurethane (PUR) polymer in dimethylformamide (range 18 - $35 \mathrm{wt} \%$ ), the most suitable spinning solution was selected.

Nanospider NS line 1WS500U from Elmarco produced nanofibrous webs using an electrostatic field. For both polymers, the distance between the electrodes was $0.142 \mathrm{~m}$; the voltage was set at $36.9 \mathrm{kV}$ and $-16.9 \mathrm{kV}$, current $0.21 \mathrm{~mA}$, the temperature $22 \pm 2{ }^{\circ} \mathrm{C}$ and the humidity $15 \pm 3 \%$ for PVB; the voltage was set at $43.7 \mathrm{kV}$ (at $0.22 \mathrm{~mA}$ ) and $-17.3 \mathrm{kV}\left(\right.$ at $0.27 \mathrm{~mA}$ ) for PUR, the temp. $25 \pm 2{ }^{\circ} \mathrm{C}$ and the humidity $17 \pm 3 \%$.

\subsection{Scanning electron microscopy}

The images were taken by the UHR FE-SEM Carl Zeiss ULTRA Plus microscope. Samples of NFs (without spunbond) were carefully cut into the squares $(5 \times 5 \mathrm{~mm})$ and attached to targets using the adhesive tape. Gold layer were applied to these samples using Quorum Q150R ES (Quorum Technologies) to ensure sufficient conductivity.
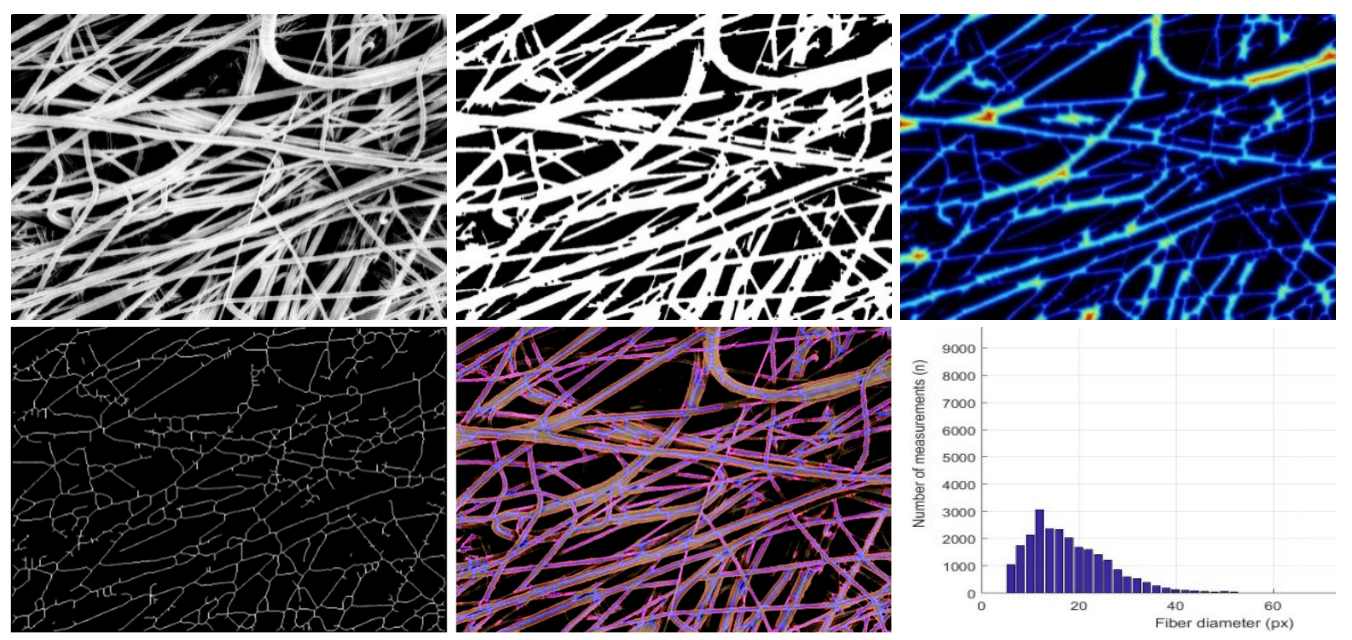

Figure 1 (a) Original image, (b) Segmented image after defects removal, (c) Distance map image,

(d) Skeleton image thinning, (e) Resulted image with detected fibers (red) and skeleton (blue),

(f) Example of histogram (fiber diameter distribution).

The image analysis was prepared within Matlab (The MathWorks, Inc.) by following steps: (1) The image of the NFs is firstly corrected through 'adapthisteq' and 'imadjust' functions. Then the image is converted from RedGreenBlue to a binary (BlackWhite) form by the Otsu algorithm ('imbinarize'). (2) Any defects (image capture errors, dust) are reduced with morphologically operation as 'bwareaopen' and 'imclose'. Porosity of NFs web is calculated as a sum of a pixel with value of " 0 " (background) to the whole picture. The pore size is determined as an 'EquivDiameter' thanks to 'regionprops' function. The equivalent circle diameter has an area equal to the area of the measured pore. (3) The thin lines of one-pixel width is calculated by algorithm called skeletonisation included in Matlab software (function 'bwmorph, skel') from the binary image. The thinning operation removes the pixels on the boundary of the object without allowing it to break apart. Total length of NFs (in each image) is calculated as a sum of all pixels in skeletonized image. (4) The distance transform is calculating with function 'bwdist, euclidean' from the binary image. The distance transform (euclidean distance) compute a sum of pixels between that pixel and the nearest nonzero pixel in binary image. (5) Images resulted 
from skeletonization and distance transform are multiplied, so only the maximum value of distance transform (where skeleton lies) are obtained. This data corresponds to fiber diameter determined in each point along the length of the fiber. The fiber linearity/curvature rating defined as the ratio of the length of a fiber that spans two selected points to the shortest distance between these two points (calculated as a minimum Feret diameter with 'bwferet, MinDiameter' function). (6) Statistical analysis is considered (average and standard deviation) for each of defined parameter. Histogram of fiber diameter and porosity distribution is plotted. The resulted image with detected fibers is imaged.

\subsection{Confocal microscopy}

Confocal microscopy was performed on the high-performance 3D optical profiler S neox from Sensofar with objective EPI 20X v35. The neox uses a high-resolution CCD (charge-coupled device, $0.69 \mu \mathrm{m} / \mathrm{pixel}$ ) sensor of up to $1360 \times 1024$ pixels (observed area $850.1 \times 709.3 \mu \mathrm{m}$ ). Samples of NFs were attached to laboratory slide using the adhesive tape. Gold layer were applied to these samples using Quorum Q150R ES (Quorum Technologies) to reduce the transparency of NFs. Surfaces can be compared in terms of roughness parameters (based on ISO 25178); including average of the height difference between five highest peaks, the five lowest valleys as "Sz" [11]; and kurtosis as "Sku" is a measuring of the sharpness of the surface height distribution.

a)
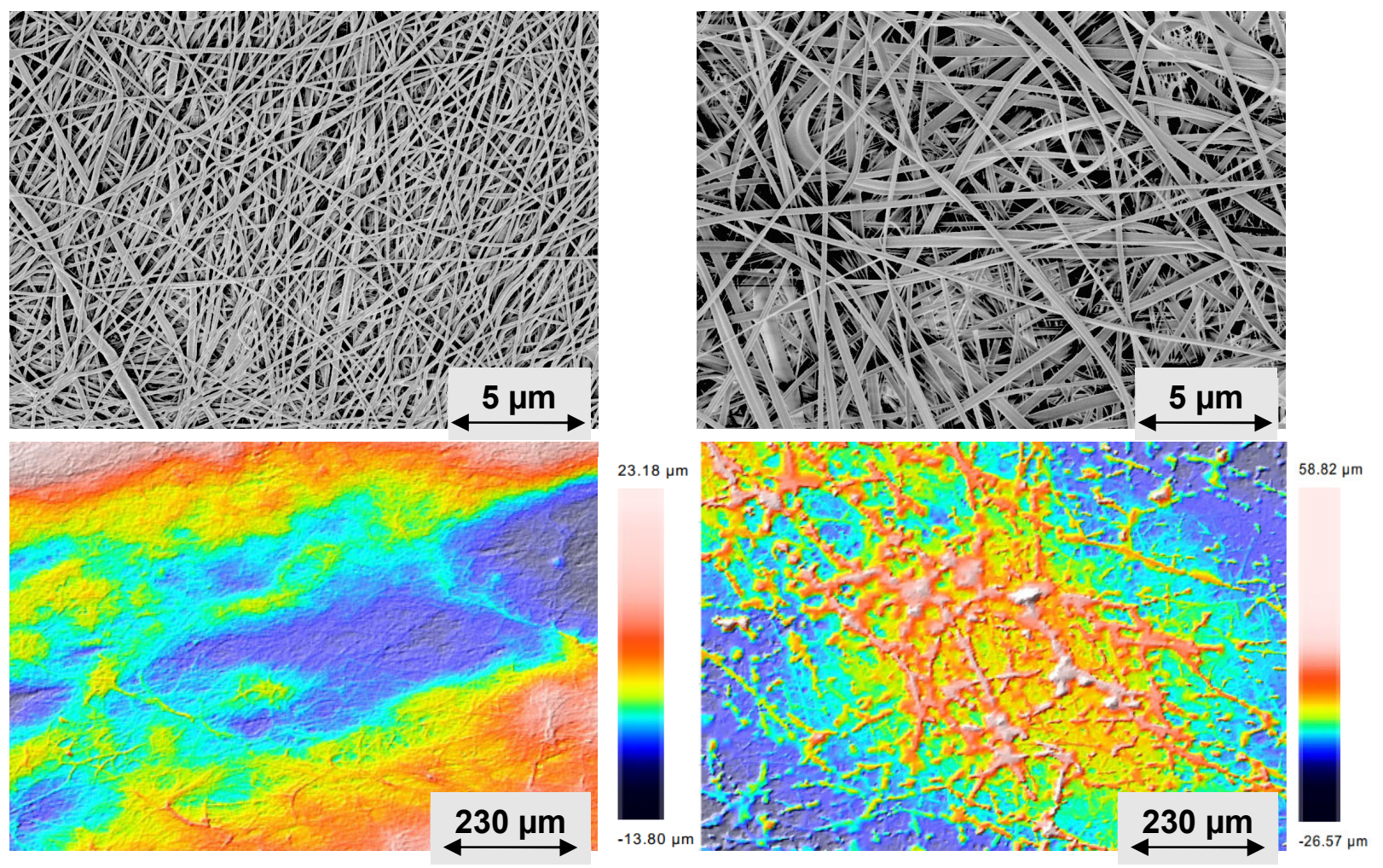

b)

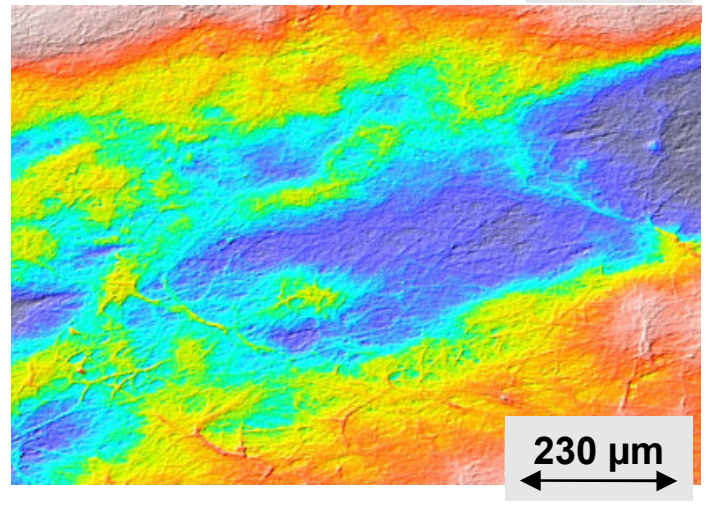

Figure 2 Images of nanofibers when Nanospider was used with (a) SEM microscopy and (b) confocal microscopy; on left are shown PUR polymer and right are PVB polymer. Note to image (b): The red to white areas in the images correspond to the upper layers of nanofibers, while the green and blue areas correspond to the lower layers of nanofibers (as shown by the color scale of the z-axis next to the images).

\subsection{Material characterization}

The biodegradability of polymeric materials is determined using a respirometric method (by measuring biochemical oxygen demand (BOD) in Micro-Oxymax Respirometer, Columbus Instruments) in the presence 
of aerobic microorganisms in an aqueous environment according to ISO 14851:1999. The samples (electrospun polymer materials) were prepared by weighing; a bacterial inoculum from real wastewater treatment plant (dry matter $4.3 \mathrm{~g} / \mathrm{L}$ and the colony forming unit value was $100000 \mathrm{CFU} / \mathrm{mL}$ ) was used. The test was carried out in standard medium (i.e. samples and inoculum were mixed with the selected volume of medium).

Differential scanning calorimetry (DSC) was performed by the DSC 6 instrument (Perkin-Elmer company) to compare the thermal transitions of the individual samples (thermo-analytical technique). Based on the results, the influence of the spinning technique on the thermal properties of polymers PVB and PUR was evaluated. The parameters glass transition $T_{g}\left({ }^{\circ} \mathrm{C}\right)$ and melting point $T_{M}\left({ }^{\circ} \mathrm{C}\right)$ were obtained.

The ball burst BB1PN was measured on device of Testometric M350-10CT. The parameters bursting strength $(\mathrm{N})$ and distance to break $(\mathrm{mm})$ were obtained.

The tensile strength was measured on device of Testometric M350-5CT. The parameters tensile strength $(\mathrm{N})$ and extension $(\mathrm{mm})$ were obtained.

\section{RESULT AND DISCUSSIONS}

Table 1 and Table 2 summarize the image analysis results and material characterization.

Table 1 Result of image analysis based on SEM and confocal microscopy

\begin{tabular}{|c|c|c|}
\hline & PUR & PVB \\
\hline Fiber length $\left(\mu \mathrm{m} / \mu \mathrm{m}^{2}\right)$ & $2.3 \pm 0.3$ & $1.4 \pm 0.2$ \\
\hline Fiber diameter $(\mathrm{nm})$ & $297 \pm 29$ & $387 \pm 42$ \\
\hline Degree of disorder (-) & $5.0 \pm 1.0$ & $4.4 \pm 0.9$ \\
\hline Porosity & $38.3 \pm 1.9$ & $44.4 \pm 1.2$ \\
\hline Pore size & $471 \pm 68$ & $697 \pm 66$ \\
\hline Roughness "Sz" $(\mu \mathrm{m})$ & $54.4 \pm 19.3$ & $75.4 \pm 6.7$ \\
\hline Kurtosis "Sku" & $4.5 \pm 1.3$ & $5.3 \pm 1.3$ \\
\hline Skewness "Ssk" (-) & $0.83 \pm 0.2$ & $1.45 \pm 0.3$ \\
\hline
\end{tabular}

Table 2 Result of material characterization based on BOD, DSC, tensile strength, and ball burst

\begin{tabular}{|c|c|c|}
\hline & PUR & PVB \\
\hline$(\mathrm{mg} / \mathrm{L})$ & $23.2 \pm 0.01$ & $22.7 \pm 0.06$ \\
\hline Glass transition $\mathrm{T}_{\mathrm{g}}\left({ }^{\circ} \mathrm{C}\right)$ & $35.9 \pm 0.03$ & $62.3 \pm 0.02$ \\
\hline Melting $T_{M}$ & $227 \pm 0.03$ & $72 \pm 0.02$ \\
\hline Tensile strength & $3.8 \pm 3.5$ & $10.7 \pm 0.5$ \\
\hline Extension & $9.2 \pm 2.3$ & $4.5 \pm 1.3$ \\
\hline Bursting strength $(\mathrm{N})$ & $9.1 \pm 4.9$ & $14.4 \pm 2.7$ \\
\hline Distance to break (mm) & $25.3 \pm 3.2$ & $32.9 \pm 2.9$ \\
\hline Angle of wettability & $88.7 \pm 12.5$ & $74.4 \pm 10.2$ \\
\hline
\end{tabular}

The biodegradability value of polymeric materials (BOD) has very little difference, thus there is unclear which morphological parameter has major impact; we can just suppose that porosity and fiber diameter will be more dominant for intensive bacterial cell colonization (material has higher active surface, thus higher number of bacteria can presence on surface).

The glass transition is influenced by fiber diameter, porosity, and roughness (higher values of fiber parameters causes higher $T_{g}$ ). The melting point is influenced by fiber length (i.e. by total amount of fiber in given area), and by degree of disorder (high disorder corresponds to higher fiber overlap and to kurtosis). Also, the correlation with BOD can be supposed (lowering melting point can led to increasing of susceptibility to enzymatic and microbial attacks and thus to higher value of BOD).

The tensile strength has a clear connection with fiber diameter and pore size; the extension is predisposed with fiber length and with degree of disorder (i.e. higher degree of disorder corresponds to more twisted or curved fibers that can be straightened, so a longer elongation can be achieved). The bursting strength has a relationship with fiber diameter, pore size and roughness; the distance to break as well with the same para- 
meters; the dominant factor can by roughness (higher roughness correspond to the distance between the lowest valley and the highest peak; higher distance correspond to higher strength that must be expended).

The angle of wettability corresponds to hydrophilic properties of surface (lower value of angle shows a better wetting; higher value applies for bad wetting hydrophobic surface). More hydrophobic material seems to be PUR polymer, which has a greater predisposition to repel water $(89 \mathrm{deg})$ than PVB $(74 \mathrm{deg})$. Only type of material used influences the interaction with water and based on image analysis method only the parameter degree of disorder seems to be in consistent with angle of wettability.

\section{CONCLUSION}

The characterization of nanofibrous materials requires a complex approach and evaluation of the results of various methods. Experimental results confirmed that mechanical properties are possible to predict by using image analysis of the surface parameters of nanofiber structures (produced by electrospinning). Detailed image analysis of fiber properties is highly important. The measuring of only fiber diameter is very underestimated evaluation. There are significant parameters that influence fibrous structures behavior (like porosity, pore size, roughness etc.) and have a significant impact on mechanical properties. The field of detailed characterization of nanomaterials can have a significant impact on creating new materials for industry and specific biological applications.

\section{ACKNOWLEDGEMENTS}

This work was supported by the Student Grant Scheme 2019 project of the Technical University in

Liberec within SGS-2019-3023 (Preparation and evaluation of nano a micro-fibrous surfaces specifically modified for the preferential growth of specific bacteria) and SGS-2019-21285 (The study and evaluation of the structure and properties of metallic and non-metallic materials).

This work was supported by the project Total Removal of Nitrogen and Phosphorus from wastewater using tailor-made textile biomass carriers (TH04030390).

\section{REFERENCES}

[1] BAHETI, S and M TUNAK. Characterization of fiber diameter using image analysis. IOP Conference Series: Materials Science and Engineering [online]. 2017, 254, 142002. ISSN 1757-8981, 1757-899X. Available from: https://doi.org/10.1088/1757-899X/254/14/142002

[2] ZIABARI, M., V. MOTTAGHITALAB and A. K. HAGHI. Application of direct tracking method for measuring electrospun nanofiber diameter. Brazilian Journal of Chemical Engineering [online]. 2009, 26(1), 53-62. ISSN 0104-6632. Available from: https://doi.org/10.1590/S0104-66322009000100006

[3] ÖZNERGIZ, Ertan, Yasar Emre KIYAK, Mustafa E. KAMASAK and Isa YILDIRIM. Automated Nanofiber Diameter Measurement in SEM Images Using and Robust Image Analysis Method. Journal of Nanomaterials [online]. 2014, 2014, 1-6. ISSN 1687-4110, 1687-4129. Available from: https://doi.org/10.1155/2014/738490

[4] ŠIRC, Jakub, Radka HOBZOVÁ, Nina KOSTINA, Marcela MUNZAROVÁ, Martina JUKLíČKOVÁ, Miloslav LHOTKA, Šárka KUBINOVÁ, Alena ZAJÍCOVÁ and Jiři MICHÁLEK. Morphological Characterization of Nanofibers: Methods and Application in Practice. Journal of Nanomaterials [online]. 2012, 2012, 1-14. ISSN 1687-4110, $1687-$ 4129. Available from: https://doi.org/10.1155/2012/327369.

[5] XU, Bugao and Yu-Liang TING. Measuring Structural Characteristics of Fiber Segments in Nonwoven Fabrics. Textile Research Journal [online]. 1995, 65(1), 41-48. ISSN 0040-5175, 1746-7748. Available from: https://doi.org/10.1177/004051759506500106

[6] SHIN, Eun Ho, Kwang Soo CHO, Moon Hwo SEO and Hyungsup KIM. Determination of electrospun fiber diameter distributions using image analysis processing. Macromolecular Research [online]. 2008, 16(4), 314-319. ISSN 1598-5032, 2092-7673. Available from: https://doi.org/10.1007/BF03218523 
[7] KRIKLAVOVA, Lucie a Tomas LEDERER. The Use of Nanofiber Carriers in Biofilm Reactor for the Treatment of Industrial Wastewaters. In: Slezska: Tanger Ltd, 2010. ISBN 978-80-87294-19-2.

[8] KRIKLAVOVA, Lucie, Tomas DUB a Tomas LEDERER. Development of Nanofiber Support for Use as a Carrier of Bacterial Biomass in Wastewater Treatment. Nanocon 2012, 4th International Conference. 2012, 460-465.

[9] SEMNANI, Dariush. A novel model of optimum nanofibre distribution in nanofibre scaffold structure by genetic algorithm method. Journal of Experimental Nanoscience [online]. 2014, 9(9), 966-981. ISSN 1745-8080, 17458099. Available from: https://doi.org/10.1080/17458080.2012.752583

[10] DING, Jianxun, Jin ZHANG, Jiannan LI, Di LI, Chunsheng XIAO, Haihua XIAO, Huanghao YANG, Xiuli ZHUANG and Xuesi CHEN. Electrospun polymer biomaterials. Progress in Polymer Science [online]. 2019, 90, 1-34. ISSN 00796700. Available from: https://doi.org/10.1016/j.progpolymsci.2019.01.002

[11] WHITEHOUSE, David J. Handbook of Surface and Nanometrology [online]. 2nd vyd. B.m.: CRC Press, 2010 [vid. 2019-04-14]. ISBN 978-1-4200-8201-2. Available from: https://www.crcpress.com/Handbook-of-Surface-andNanometrology-Second-Edition/Whitehouse/p/book/9781420082012 